\title{
Measuring Higgs Couplings without Higgs Bosons
}

\author{
Brian Henning, Davide Lombardo, Marc Riembau, and Francesco Riva \\ Départment de Physique Théorique, Université de Genève, 24 quai Ernest-Ansermet, 1211 Genève 4, Switzerland
}

(Received 8 February 2019; revised manuscript received 2 July 2019; published 29 October 2019)

\begin{abstract}
The measurement of Higgs couplings constitutes an important part of present standard model precision tests at colliders. We show that modifications of Higgs couplings induce energy-growing effects in specific amplitudes involving longitudinally polarized vector bosons, and we initiate a novel program to study these effects off shell and at high energy, rather than on the Higgs resonance. Our analysis suggests that these channels are complementary and competitive with familiar on-shell measurements; moreover, they offer endless opportunities for refinement and improvements.
\end{abstract}

DOI: 10.1103/PhysRevLett.123.181801

The precise measurement of the Higgs boson couplings to other standard model (SM) particles is an unquestionable priority in the future of particle physics. These measurements are important probes for our understanding of a relatively poorly measured sector of the SM; at the same time they offer a window into heavy dynamics beyond the standard model (BSM). Indeed, it is well known that the exchange of heavy states (with masses beyond the direct collider reach) leaves imprints in low-energy experiments, in a way that is systematically captured by an effective field theory (EFT).

In processes in which a Higgs boson is produced on shell, the narrow width approximation allows one to parametrize departures from the SM as modified rates of the Higgs boson to the SM particles. For this reason, the well-established method for testing Higgs couplings (HC) is to measure resonant Higgs boson processes.

In this Letter we initiate a novel program to test the very same Higgs couplings, off shell and at high energy, via their contributions to the physics of longitudinally polarized gauge bosons. We identify a series of such processes, where the effect of modified $\mathrm{HC}$ grows with the energy $E$ : for $E^{2}$ growing effects, a $1 \%$ sensitivity at the Higgs boson mass corresponds, in principle, to a $\mathrm{O}(1)$ sensitivity at $E \sim 1 \mathrm{TeV}$. While $E$-growing effects are widely discussed in the literature, here we extend the high-energy program to all Higgs couplings and show that it can potentially be competitive with on-shell measurements.

Moreover-and perhaps most importantly - this program contains numerous avenues for refinement and improvements: it can benefit maximally from accumulated statistics,

Published by the American Physical Society under the terms of the Creative Commons Attribution 4.0 International license. Further distribution of this work must maintain attribution to the author(s) and the published article's title, journal citation, and DOI. Funded by SCOAP ${ }^{3}$. from improved SM computations of differential distributions, from phenomenological analyses aimed at enhancing the signal-over-background (see, for instance, Refs. [1-6]), and from dedicated experimental analyses. Furthermore, given the complexity of the final states, we expect that advanced machine learning techniques [7-9] could drastically improve our simple cut and count analysis. Additionally, in the context of a global precision program, the high-energy aspects discussed here will benefit the most, not only from the long-term high-luminosity (HL) LHC program [10], but also from potential future high-energy colliders, such as the high-energy LHC [10] or CLIC [11].

Our leitmotiv is that any observable modification of a SM coupling will produce in some process a growth with energy (see Table I). In some sense, this is obvious: since the SM is the only theory that can be extrapolated to parametrically high energy, any departure from it can have only a finite range of validity, a fact that is made manifest by a disproportionate growth in some scattering amplitude. Theories with a finite range of validity are, by definition, EFTs.

HCs are associated with an EFT Lagrangian $\mathcal{L}=$ $\sum_{i} c_{i} \mathcal{O}_{i} / \Lambda^{2}$, consisting in particular of the dimensionsix operators $[12,13]$,

$$
\begin{aligned}
\mathcal{O}_{r} & =|H|^{2} \partial_{\mu} H^{\dagger} \partial^{\mu} H, & & \mathcal{O}_{y_{\psi}}=Y_{\psi}|H|^{2} \psi_{L} H \psi_{R}, \\
\mathcal{O}_{B B} & =g^{\prime 2}|H|^{2} B_{\mu \nu} B^{\mu \nu}, & & \mathcal{O}_{W W}=g^{2}|H|^{2} W_{\mu \nu}^{a} W^{a \mu \nu}, \\
\mathcal{O}_{G G} & =g_{s}^{2}|H|^{2} G_{\mu \nu}^{a} G^{a \mu \nu}, & & \mathcal{O}_{6}=|H|^{6},
\end{aligned}
$$

with $Y_{\psi}$ the Yukawa coupling for the fermion $\psi$. [Note that the parameters in Eq. (3) can be put in correspondence with other parametrizations of HCs: via partial widths $\kappa_{i}^{2}=$ $\Gamma_{h \rightarrow i i} / \Gamma_{h \rightarrow i i}^{\mathrm{SM}}[14]$, via Lagrangian couplings in the unitary gauge $g_{\text {hii }}[13,15]$, or via pseudo-observables [16].]

The operators of Eq. (1) have the form $|H|^{2} \times \mathcal{O}^{\mathrm{SM}}$, with $\mathcal{O}^{\mathrm{SM}}$ a dimension-four SM operator (i.e., kinetic terms, Higgs potential, and Yukawa couplings) times 
TABLE I. Each effect (left-hand column) can be measured as an on-shell Higgs coupling (diagram in the HC column) or in a highenergy process (diagram in the $\mathrm{HwH}$ column), where it grows with energy as indicated in the last column.

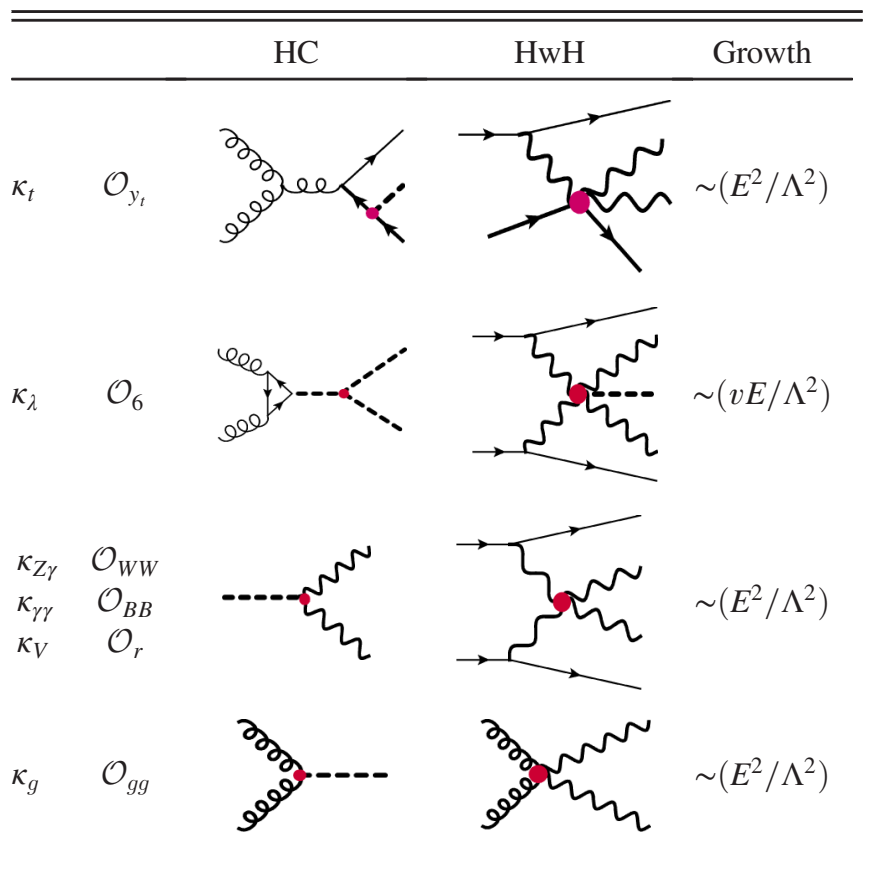

$$
|H|^{2}=\frac{1}{2}\left[v^{2}+2 h v+h^{2}+2 \phi^{+} \phi^{-}+\left(\phi^{0}\right)^{2}\right],
$$

where $v=246 \mathrm{GeV}$ is the Higgs vacuum expectation value, $h$ is the physical Higgs boson, and $\phi^{ \pm, 0}$ are the would-be longitudinal polarizations of $W$ and $Z$ bosons. From the operators in Eq. (1), the piece $\propto v^{2}$ can be reabsorbed via a redefinition of the SM input parameters and is therefore unobservable [17,18]; the piece $\propto v h$ constitutes instead the core of the $\mathrm{HC}$ measurements program, as it implies modifications to single-Higgs processes (triple Higgs processes for $\mathcal{O}_{6}$ ), and can be matched easily to the $\kappa$ framework. The $h^{2}$ piece was discussed in Refs. [19-21] in the context of double-Higgs production. In this Letter we focus on the last two terms in Eq. (2) and study processes with longitudinal gauge bosons instead of processes with an on-shell Higgs boson; we dub this search strategy "Higgs without Higgs"- $\mathrm{HwH}$ in short.

The first ingredient in this program is to identify which processes grow maximally with energy once Higgs couplings are modified. There is a quick and intuitive way to assess this based on (1) dimensional analysis, (2) our choice of EFT basis Eq. (1), and (3) the parametrization chosen in Eq. (2), where the longitudinal polarizations are explicitly represented by their scalar high-energy counterpart [22-24]. For $v \rightarrow 0$, the operators of Eq. (1) contribute directly to contact interactions with $n=4$ fields $\left(\mathcal{O}_{W W}, \mathcal{O}_{B B}, \mathcal{O}_{G G}, \mathcal{O}_{r}\right), 5$ fields $\left(\mathcal{O}_{y_{\psi}}\right)$, or 6 fields $\left(\mathcal{O}_{6}\right)$, with a coupling $\propto 1 / \Lambda^{2}$ that carries two inverse powers of mass dimensions. At high-energy $\left(E \gg m_{W, h, t}\right)$, dimensional analysis implies that amplitudes generated by these contact vertices are maximally energy growing; therefore, generically, we expect that the BSM and SM contributions scale as

$$
\frac{\mathcal{A}_{n}^{\mathcal{O}}}{\mathcal{A}_{n}^{\mathrm{SM}}} \sim \frac{E^{2}}{\Lambda^{2}}
$$

Table I shows the relevant processes that exhibit this behavior; more explicitly, at hadron (lepton) colliders,

$$
\begin{array}{cc}
\kappa_{t}: p p \rightarrow j t+V_{L} V_{L}^{\prime}, & \\
\left(e^{+} e^{-} \rightarrow l l+\left\{t b W_{L}, t b Z_{L}, t t W_{L}, t t Z_{L}\right\}\right), \\
\kappa_{\lambda}: p p \rightarrow j j h+V_{L} V_{L}^{\prime}, & \left(e^{+} e^{-} \rightarrow l l h V_{L} V_{L}^{\prime}\right), \\
p p \rightarrow j j+4 V_{L}, & \left(e^{+} e^{-} \rightarrow l l 4 V_{L}\right), \\
\kappa_{\gamma \gamma, Z \gamma}: p p \rightarrow j j+V^{\prime} V, & \left(e^{+} e^{-} \rightarrow l l V^{\prime} V\right), \\
\kappa_{V}: p p \rightarrow j j+V_{L} V_{L}^{\prime}, & \left(e^{+} e^{-} \rightarrow l l V_{L} V_{L}^{\prime}\right), \\
\kappa_{g}: p p \rightarrow W_{L}^{+} W_{L}^{-}, Z_{L} Z_{L}, & \left(e^{+} e^{-} \rightarrow l l j j\right),
\end{array}
$$

where $V_{L} V_{L}^{\prime} \equiv\left\{W_{L}^{ \pm} W_{L}^{ \pm}, W_{L}^{ \pm} W_{L}^{\mp}, W_{L}^{ \pm} Z_{L}, Z_{L} Z_{L}\right\} \quad$ (similarly $4 V_{L}$ is a generic longitudinally polarized final state) and $V^{(\prime)}$ is any (longitudinal or transverse) vector, including photons, while $l$ denotes either a charged lepton $\ell^{ \pm}$or a neutrino, depending on the final state. Notice that the amplitude associated with the modified couplings grows quadratically with energy $E^{2}$ [with the exception of Eq. (5), see below].

In the following paragraphs we explore these processes in turn and provide a first estimate of the potential $\mathrm{HwH}$ reach at the HL-LHC in comparison with the reach from Higgs couplings measurements. Our results are based on leading order MadGraph simulations [25], where the Higgs couplings have been modified using FeynRules [8] and checked against the model of Ref. [26].

Top Yukawa coupling.-Modifications of the Yukawa coupling of the Higgs boson to top quarks is reputedly difficult to measure on the $h$ resonance [27]; however, according to the above discussion, an anomalous top quark Yukawa coupling induces a quadratic energy growth in the five point amplitude $\mathcal{A}(b V \rightarrow t V V)$. This amplitude leads to a process with a final state consisting of a top quark, a forward jet, and two longitudinally polarized vector bosons in the final state; see Eq. (4). Notice that these have a smaller energy threshold compared with the $t$ th final state used in HC measurements. (See also Ref. [28] that studies thj final states which exhibits linear $E$ growth with modifications of the top Yukawa coupling.) 
The top carries a large transverse momentum $p_{T}^{t}$ due to the hardness of the process, which makes it a good discriminator. We consider two categories, for $p_{T}^{t}>250(500) \mathrm{GeV}$. A forward jet with $\left|\eta_{j}\right|>2.5$, $p_{T}^{j}>30 \mathrm{GeV}$, and $E_{j}>300 \mathrm{GeV}$ is required.

The signal is classified by counting the number of extra leptons reconstructed in the event. The following table shows the number of signal events at the $14 \mathrm{TeV}$ HL-LHC with $3000 \mathrm{fb}^{-1}$, for $p_{T}^{t}>250 / 500 \mathrm{GeV}$.

\begin{tabular}{lccccc}
\hline Process & $0 \ell$ & $1 \ell$ & $\ell^{ \pm} \ell^{\mp}$ & $\ell^{ \pm} \ell^{ \pm}$ & $3 \ell(4 \ell)$ \\
\hline$W^{ \pm} W^{\mp}$ & $3449 / 567$ & $1724 / 283$ & $216 / 35$ & $\ldots$ & $\cdots$ \\
$W^{ \pm} W^{ \pm}$ & $2850 / 398$ & $1425 / 199$ & $\cdots$ & $178 / 25$ & $\cdots$ \\
$W^{ \pm} Z$ & $3860 / 632$ & $965 / 158$ & $273 / 45$ & $\ldots$ & $68 / 11$ \\
$Z Z$ & $2484 / 364$ & $\cdots$ & $351 / 49$ & $\ldots$ & $(12 / 2)$
\end{tabular}

The categories with two or more leptons have small background. For the hadronic modes-which dominate the 0 and 1 lepton channels - the largest source of background comes from $\bar{t} t j j \rightarrow t W b j j$, where the $b$ quark gets misidentified as an ordinary jet and the two lowest rapidity jets reconstruct a $W / Z$ boson. After applying the event topology selection cuts - the required forward jet, the invariant mass of the two lower rapidity jets reconstructs an electroweak gauge boson mass, and a boosted top-the cross section is $470 \mathrm{fb}(22 \mathrm{fb})$ for $p_{T}^{t}>250 \mathrm{GeV}$ $(>500 \mathrm{GeV}$ ), roughly 80 (20) times that of the signal. However, in order to fall into the signal region, the $b$ quark must be misidentified as a regular jet and the pair of lower rapidity jets must mimic a hadronically decaying vector. The $b$ misidentification rate is order $10 \%$ for a $90 \%$ light jet acceptance [29]. Vector boson tagging techniques [30] can identify a hadronically decaying vector with a $10^{2}$ background rejection for a $40 \%$ signal efficiency.
A conservative estimate of the combined effect of these cuts brings the background to comparable or smaller size than the signal.

We broadly parametrize this and other backgrounds by a uniform rescaling $B$ of the SM signal expectation in each bin (so that for $B=1$ we add an irreducible background equal to the SM signal in each channel), and show the estimated reach in the left-hand panel of Fig. 1. The dashed gray lines compare our results with those from $\mathrm{HC}$ measurements [10]. The large number of events left in the zero and one lepton categories makes it possible to extend the analysis to higher energies, where not only the effects of the energy growth will be enhanced, but also the background reduced; a dedicated study is needed to assess more precisely the acceptances of these hadronic channels.

This mode of exploration also appears well suited for high-energy lepton colliders like CLIC. Indeed, the processes in the second line of Eq. (4) have a lower threshold for production than the $\bar{t} t h$ final state that is usually considered to measure the top quark Yukawa.

Higgs boson self-coupling.-Measurements of the Higgs boson self-coupling have received enormous attention in collider studies. In the di-Higgs channel at HL-LHC, the Higgs boson self-coupling modification $\delta \kappa_{\lambda}$ is expected to be constrained within $[-1.8,6.7]$ at $95 \%$ C.L. [31] using the $b \bar{b} \gamma \gamma$ final state. Here we propose the processes of Eqs. (5) ad (6) with vector boson scattering (VBS) topology and a multitude of longitudinally polarized vector boson; see, e.g., the second row of Table I. The modified coupling $\delta \kappa_{\lambda}$, or the operator $\mathcal{O}_{6}$, induces a linear growth with energy with respect to the $\mathrm{SM}$ in processes with $j j h V_{L} V_{L}$ final state (Table I), and a quadratic growth in processes with $j j V_{L} V_{L} V_{L} V_{L}$. For the former, the same-sign $W^{ \pm} W^{ \pm} h j j$ with leptonic $(e, \mu)$ decays is particularly favorable for its low background: two same-sign leptons and VBS topology
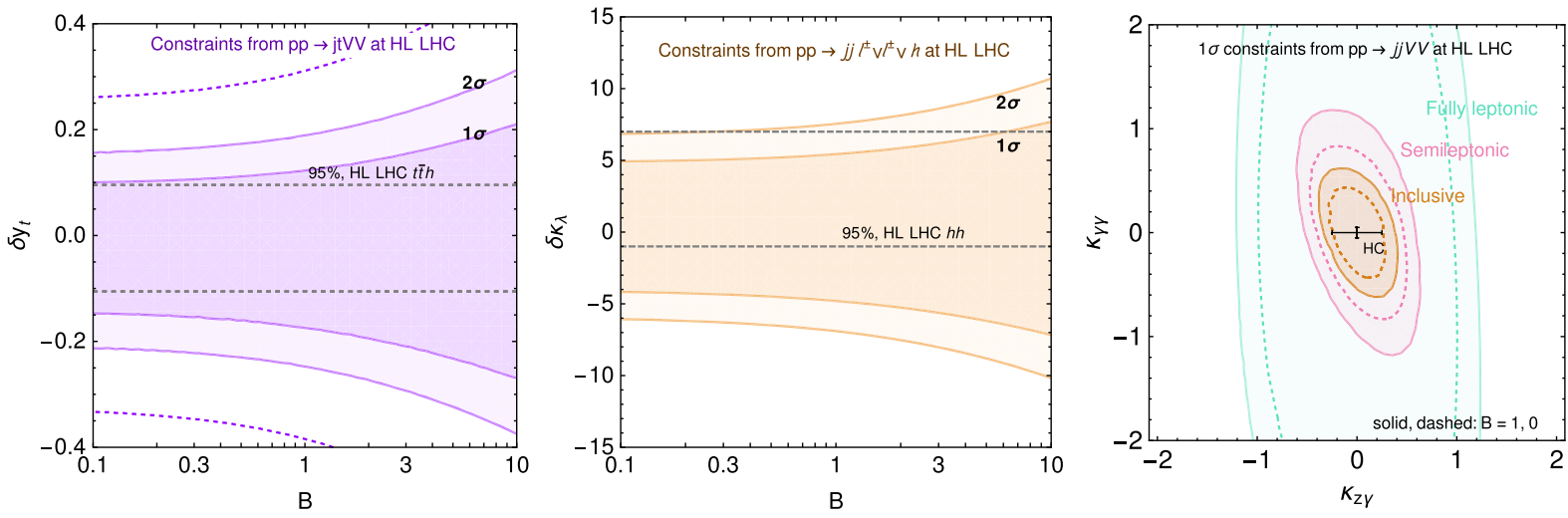

FIG. 1. Left: HL-LHC (3000 $\left.\mathrm{fb}^{-1}\right)$ sensitivity on modifications of the top quark Yukawa coupling $\delta y_{t}$ from the process in Eq. (4) (shaded bands), and from measurements of Higgs couplings (95\% C.L., dashed gray lines); $B$ controls additional backgrounds (for $B=1$ the analysis includes a number of background events equal to the SM signal); the dashed purple line corresponds to $1 \sigma$ results for the $\geq 2 \ell$ categories (which have the smallest backgrounds). Center: Same but for modifications of the Higgs trilinear $\delta \kappa_{\lambda}$. Right: $1 \sigma$ reach for modification of the Higgs- $\gamma \gamma$ and $Z \gamma$ rates, using high- $E$ measurements (green, pink, and brown bands correspond to leptonic, semileptonic, and also hadronic final states) or Higgs couplings (black error bars). 
offers a good discriminator against background, allowing for $h \rightarrow \bar{b} b$ decays. For illustration we focus on this channel in which the SM gives $N_{\mathrm{SM}} \simeq 50$ events. Backgrounds from $t \bar{t} j j$ enter with a misidentified lepton, but it can be shown that they can be kept under control with the efficiencies reported in Ref. [32] and with VBS cuts on the forward jets. A potentially larger background is expected to come from fake leptons, but the precise estimation of it is left for future work.

The results — shown in the center panel of Fig. 1-are very encouraging: this simple analysis can match the precision of the by-now very elaborate di-Higgs studies. There are many directions in which this approach can be further refined: (i) including the many other final states in Eq. (5), both for the vector decays and for the Higgs decay, (ii) including the $E^{2}$-growing $j j V_{L} V_{L} V_{L} V_{L}$ topologies of Eq. (6), or (iii) taking into account differential information. Moreover, the process of Table I grows only linearly with energy with respect to the SM amplitude with transverse vectors in the final state, but it grows quadratically with respect to the SM longitudinal final states, so (iv) measurements of the polarization fraction can improve this measurement.

Higgs boson to $\gamma \gamma, Z \gamma$.-These decay rates are loop level and small in the SM: their measurement implies therefore tight constraints on possible large (tree-level) BSM effects, which in the EFT language are captured by the operators $\mathcal{O}_{W W, B B}$ from Eq. (1). These also enter in high-energy VBS Eq. (7), and they represent a beautiful additional motivation (together with $\kappa_{V}$, see below) to study these processes, which at present are often interpreted in the context of anomalous quartic gauge couplings (aQGCs) [33], corresponding to dimension-eight operators.

We perform a simple analysis of vector boson scattering with $W^{ \pm} W^{ \pm}, Z Z, W Z, Z \gamma$ final states. For the first three we cut as usual on the forward jets: $\left|\delta_{j j}\right|>2.5, p_{T}^{j}>30 \mathrm{GeV}$, and $m_{j j}>500 \mathrm{GeV}$ [34]. A kinematic variable that captures the hardness of the $2 \rightarrow 2$ process is the scalar sum of the $p_{T}^{V}$ of the vector bosons, and therefore we divide the distribution in bins of $250 \mathrm{GeV}$ up to $2 \mathrm{TeV}$. For the $Z \gamma$ final state, we follow the analysis for aQGC of Ref. [35].

The combined results are displayed in the right-hand panel of Fig. 1, for fully leptonic, semileptonic, and fully hadronic decays (a difficult challenge at the LHC), for backgrounds $B=0,1$ where, as explained above, $B=1$ corresponds to an additional background of the same order as the SM. Note that we translated constraints on $c_{B B}, c_{W W}$ to $\kappa_{\gamma \gamma}, \kappa_{z \gamma}$. We find that the $Z Z, Z \gamma$ final states provide the best reach. For comparison, the individual reach from HL-LHC measurements of HCs [10] is shown by the black error bars. These clearly offer an unbeatable sensitivity in the $h \gamma \gamma$ direction; the $h Z \gamma$ direction is, however, less tested, and our simple analysis of high-energy probes shows promising results.

Higgs boson to $W^{+} W^{-}, Z Z$. - It is known that modifications of the tree-level $h Z Z$ and $h W^{+} W^{-}$SM couplings (assumed here to be controlled by a unique parameter, corresponding for instance to $\mathcal{O}_{r}$ ) imply a quadratic $E$ growth in longitudinal VBS. This is discussed in detail in Ref. [36] (and in Ref. [37] for linear colliders), where it is pointed out that, in the SM, the longitudinal component is suppressed by an accidental factor 2000, which is equivalent to a very large irreducible background. This motivated studies of VBS $h h$ pair production instead, see Ref. [20], finding at $1 \sigma, \delta \kappa_{V} \lesssim 8 \%$, comparable to $\delta \kappa_{V} \lesssim$ $5 \%$ from HC [10]. (The numbers we report here are indicative: both analyses have optimistic and pessimistic scenarios in which these numbers might differ.)

Higgs boson to gg.-This coupling modifies the main production mode at hadron colliders and is, therefore, very well measured. The most interesting high-energy process that can be associated with this coupling is $g g \rightarrow Z Z$, which has been discussed in Refs. [38-40]. Using the results from Ref. [38] we estimate $\mathrm{HwH}$ versus $\mathrm{HC}$ reach at the end of the HL-LHC,

$\mathrm{HC}:\left|\delta \kappa_{g}\right| \lesssim 0.025$,

HwH: $\left|\delta \kappa_{g}\right| \lesssim 0.24 / 0.06 / 0.01$,

$\mathrm{HwH}:\left(\right.$ no $\left.\bar{q} q \rightarrow Z_{T} Z_{T}\right):\left|\delta \kappa_{g}\right| \lesssim 0.09 / 0.02 / 0.005$,

where the numbers stand for the fully leptonic, semileptonic, fully hadronic channels. The partonic $\bar{q} q \rightarrow Z_{T} Z_{T}$ process represents here the main irreducible background, as it does not interfere with our $g g \rightarrow Z_{L} Z_{L}$ amplitude with longitudinal polarization. Its reduction would constitute an important aspect of $\mathrm{HwH}$ analyses, as shown by the results in the third line of Eq. (10). Notice that, unfortunately, in the SM the $g g \rightarrow Z_{L} Z_{L}$ process is extremely suppressed at high $E$, to the benefit of the transverse $T T$ process; see Ref. [41]. This implies that the SM-BSM interference is also suppressed.

Despite these difficulties, which might be overcome in more refined analyses (along the lines of Refs. [4,5]), the high- $E$ results are potentially interesting in the semileptonic and fully hadronic channels.

The amplitude we propose can also find a beautiful implementation in the context of future lepton colliders, in particular ILC and CLIC, in the form of ZZ,WW $\rightarrow g g$ in VBS. There, the possibility to polarize the initial electron positron beams could offer an additional handle to enhance the longitudinal polarizations.

Discussion. - Any deviations from the SM predictions of the processes considered imply an ultimate cutoff of the theory: the scale of unitarity violation $\Lambda_{\mathrm{sc}}$ where the particles of the SM would become strongly coupled in the absence of new dynamics. The possibility of a consistent EFT interpretation requires $E<\Lambda_{\text {sc }}$; see, e.g., Refs. [42-44]. $\Lambda_{\mathrm{sc}}$ depends on the size of the deviations; for instance, for $\mathrm{O}(1)$ deviations from the Higgs boson selfcoupling $\kappa_{\lambda}$, this scale is of order $13 \mathrm{TeV}[45,46]$, much larger than the typical relevant energy that we are accessing 
at the LHC. Other Higgs couplings are better measured and hence have larger strong-coupling scales associated, and therefore a wider EFT validity range. These considerations imply that our analysis - which utilizes high-energy bins of differential distributions-admit a consistent EFT treatment.

There are many directions in which our analysis can be extended and refined, and which we will investigate in future work. (1) Realistic estimates of the relevant backgrounds and acceptances in the different channels are the first step in the HwH program; this motivates the development of tools, along the lines of Ref. [30], to reject QCD background from a hadronically decaying vector-boson signal. (2) Moreover, our signals center on the presence of longitudinally polarized vector bosons. An important irreducible background in this context is the SM fraction of transversely polarized states which, in many cases, is much larger than the longitudinal signal [36]. So, important progress could come from a better understanding of the kinematics of the various helicity amplitudes, aimed at improving the signal-to-background ratio, along the lines of Refs. [4,5,47], or other techniques that access information about vector-boson polarization [48-50]. (3) A more refined understanding of the relevant scales and BSMsensitive distributions in the problem, as in Ref. [28], or complemented with more advanced boosted decision tree or machine learning techniques, see, e.g., Refs. [7-9], would also increase $\mathrm{HwH}$ sensitivity. (4) A realistic analysis would also include QCD corrections; these tend to increase the relevant cross sections, but also complicate their helicity structure. (5) It would be interesting to include our observables in the context of a global fit (see, e.g., Refs. [17,51-54]): we expect that, even in situations where they cannot directly compete with individual HC measurements, they can still provide valuable global information to access potential flat directions. (6) Finally, if the Higgs boson is not part of a doublet or electroweak symmetry breaking effects are large (i.e., a nonlinear realization of electroweak symmetry with a singlet, also known as HEFT), then contributions to the processes in Eqs. (4)(9) from operators involving only the longitudinal polarizations will be decorrelated from the operators involving only the physical Higgs bosons entering $\mathrm{HC}$ processes, thus potentially offering an opportunity to distinguish between HEFT and SM EFT.

We thank Pietro Govoni, Matthew McCullough, Johnny Raine, and Steven Schramm for valuable discussions. This project is funded by the Swiss National Science Foundation under Grant No. PP002-170578.

[1] M. Farina, G. Panico, D. Pappadopulo, J. T. Ruderman, R. Torre, and A. Wulzer, Phys. Lett. B 772, 210 (2017).

[2] R. Franceschini, G. Panico, A. Pomarol, F. Riva, and A. Wulzer, J. High Energy Phys. 02 (2018) 111.
[3] S. Alioli, M. Farina, D. Pappadopulo, and J. T. Ruderman, J. High Energy Phys. 07 (2017) 097.

[4] A. Azatov, J. Elias-Miro, Y. Reyimuaji, and E. Venturini, J. High Energy Phys. 10 (2017) 027.

[5] G. Panico, F. Riva, and A. Wulzer, Phys. Lett. B 776, 473 (2018).

[6] S. Banerjee, C. Englert, R. S. Gupta, and M. Spannowsky, Phys. Rev. D 98, 095012 (2018).

[7] J. Brehmer, K. Cranmer, G. Louppe, and J. Pavez, Phys. Rev. Lett. 121, 111801 (2018).

[8] N. D. Christensen and C. Duhr, Comput. Phys. Commun. 180, 1614 (2009).

[9] R. T. D'Agnolo and A. Wulzer, Phys. Rev. D 99, 015014 (2019).

[10] M. Cepeda et al., arXiv:1902.00134.

[11] J. de Blas et al., The CLIC potential for new physics, in CERN Yellow Reports: Monographs Vol. 3 (CERN, Geneva, Switzerland, 2018).

[12] B. Grzadkowski, M. Iskrzynski, M. Misiak, and J. Rosiek, J. High Energy Phys. 10 (2010) 085.

[13] D. de Florian et al. (LHC Higgs Cross Section Working Group), Handbook of LHC Higgs cross sections: 4. Deciphering the nature of the Higgs sector, in CERN Yellow Reports: Monographs Vol. 2 (CERN, Geneva, Switzerland, 2017).

[14] J. R. Andersen et al. (LHC Higgs Cross Section Working Group), CERN Reports No. CERN-2013-004 and FERMILAB-CONF-13-667-T, 2013, 10.5170/CERN-2013-004.

[15] R. S. Gupta, A. Pomarol, and F. Riva, Phys. Rev. D 91, 035001 (2015).

[16] M. Gonzalez-Alonso, A. Greljo, G. Isidori, and D. Marzocca, Eur. Phys. J. C 75, 128 (2015).

[17] A. Pomarol and F. Riva, J. High Energy Phys. 01 (2014) 151.

[18] J. Elias-Miro, J. Espinosa, E. Masso, and A. Pomarol, J. High Energy Phys. 11 (2013) 066.

[19] F. Goertz, A. Papaefstathiou, L. L. Yang, and J. Zurita, J. High Energy Phys. 04 (2015) 167.

[20] F. Bishara, R. Contino, and J. Rojo, Eur. Phys. J. C 77, 481 (2017).

[21] A. Azatov, R. Contino, G. Panico, and M. Son, Phys. Rev. D 92, 035001 (2015).

[22] J. M. Cornwall, D. N. Levin, and G. Tiktopoulos, Phys. Rev. D 10, 1145 (1974); 11, 972(E) (1975).

[23] M. S. Chanowitz and M. K. Gaillard, Nucl. Phys. B261, 379 (1985).

[24] A. Wulzer, Nucl. Phys. B885, 97 (2014).

[25] J. Alwall, R. Frederix, S. Frixione, V. Hirschi, F. Maltoni, O. Mattelaer, H. S. Shao, T. Stelzer, P. Torrielli, and M. Zaro, J. High Energy Phys. 07 (2014) 079.

[26] A. Falkowski, B. Fuks, K. Mawatari, K. Mimasu, F. Riva, and V. Sanz, Eur. Phys. J. C 75, 583 (2015).

[27] M. Aaboud et al. (ATLAS Collaboration), Phys. Lett. B 784, 173 (2018).

[28] C. Degrande, F. Maltoni, K. Mimasu, E. Vryonidou, and C. Zhang, J. High Energy Phys. 10 (2018) 005.

[29] ATLAS Collaboration, CERN Report No. ATLAS-PHYSPUB-2017-013, 2017.

[30] ATLAS Collaboration, CERN Report No. ATLAS-CONF2018-016, 2018. 
[31] ATLAS Collaboration, CERN Report No. ATL-PHYSPUB-2017-001, 2017.

[32] V. Khachatryan et al. (CMS Collaboration), J. Instrum. 10, P06005 (2015).

[33] O. J. P. Eboli, M. C. Gonzalez-Garcia, and J. K. Mizukoshi, Phys. Rev. D 74, 073005 (2006).

[34] G. Aad et al. (ATLAS Collaboration), Phys. Rev. Lett. 113, 141803 (2014).

[35] M. Aaboud et al. (ATLAS Collaboration), J. High Energy Phys. 07 (2017) 107.

[36] R. Contino, C. Grojean, M. Moretti, F. Piccinini, and R. Rattazzi, J. High Energy Phys. 05 (2010) 089.

[37] R. Contino, C. Grojean, D. Pappadopulo, R. Rattazzi, and A. Thamm, J. High Energy Phys. 02 (2014) 006.

[38] A. Azatov, C. Grojean, A. Paul, and E. Salvioni, Zh. Eksp. Teor. Fiz. 147, 410 (2015) [J. Exp. Theor. Phys. 120, 354 (2015)].

[39] G. Cacciapaglia, A. Deandrea, G. D. La Rochelle, and J.-B. Flament, Phys. Rev. Lett. 113, 201802 (2014).

[40] A. Azatov, C. Grojean, A. Paul, and E. Salvioni, J. High Energy Phys. 09 (2016) 123.

[41] E. W. N. Glover and J. J. van der Bij, Nucl. Phys. B321, 561 (1989).
[42] A. Biekötter, A. Knochel, M. Krämer, D. Liu, and F. Riva, Phys. Rev. D 91, 055029 (2015).

[43] R. Contino, A. Falkowski, F. Goertz, C. Grojean, and F. Riva, J. High Energy Phys. 07 (2016) 144.

[44] F. Pobbe, A. Wulzer, and M. Zanetti, J. High Energy Phys. 08 (2017) 074.

[45] S. Chang and M. A. Luty, arXiv:1902.05556.

[46] A. Falkowski and R. Rattazzi, arXiv:1902.05936.

[47] L. J. Dixon and Y. Shadmi, Nucl. Phys. B423, 3 (1994); B452, 724(E) (1995).

[48] CMS Collaboration, CERN Report No. CMS-PAS-FTR-18005, 2018.

[49] CMS Collaboration, CERN Report No. CMS-PAS-FTR-18014, 2018.

[50] J. Lee, N. Chanon, A. Levin, J. Li, M. Lu, Q. Li, and Y. Mao, Phys. Rev. D 99, 033004 (2019).

[51] R. Gómez-Ambrosio, Eur. Phys. J. C 79, 389 (2019).

[52] A. Biekötter, T. Corbett, and T. Plehn, SciPost Phys. 6, 064 (2019).

[53] J. de Blas, M. Ciuchini, E. Franco, S. Mishima, M. Pierini, L. Reina, and L. Silvestrini, J. High Energy Phys. 12 (2016) 135.

[54] J. Ellis, C. W. Murphy, V. Sanz, and T. You, J. High Energy Phys. 06 (2018) 146. 